REVUE DE L'INSTITUT

FRANÇAIS D'HISTOIRE

EN ALLEMAGNE

\section{Revue de l'IFHA}

Revue de l'Institut français d'histoire en Allemagne

\title{
Retours sur un projet de réforme. À propos de l'ouvrage collectif Was war Bielefeld? Eine ideengeschichtliche Nachfrage
}

Jochen Hoock

\section{(2) OpenEdition}

\section{Journals}

Édition électronique

URL : http://journals.openedition.org/ifha/192

DOI : $10.4000 /$ ifha. 192

ISSN : 2198-8943

Éditeur

IFRA - Institut franco-allemand (sciences historiques et sociales)

\section{Édition imprimée}

Date de publication : 6 février 2011

ISSN : 2190-0078

\section{Référence électronique}

Jochen Hoock, « Retours sur un projet de réforme. À propos de l'ouvrage collectif Was war Bielefeld? Eine ideengeschichtliche Nachfrage », Revue de I'IFHA [En ligne], 3 | 2011, mis en ligne le 19 avril 2012 consulté le 21 avril 2019. URL : http://journals.openedition.org/ifha/192 ; DOI : 10.4000/ifha.192

Ce document a été généré automatiquement le 21 avril 2019

(CIFHA 


\title{
Retours sur un projet de réforme. À propos de l'ouvrage collectif Was war Bielefeld? Eine ideengeschichtliche Nachfrage
}

\author{
Jochen Hoock
}

\section{NOTE DE L'ÉDITEUR}

Jochen Hoock est professeur émérite à l'université Paris VII-Denis-Diderot.

L'ouvrage dont il est question est celui de Sonja Asal, Stephan Schlak (dir.), Was war Bielefeld? Eine ideengeschichtliche Nachfrage, Göttingen: Wallstein (Marbacher Schriften, Neue Folge, 4), 2009.

La crise des institutions universitaires incite à se pencher sur le sort des grands projets de réforme issus de la vague de créations universitaires de la fin des années 1960 et des années 1970. C'est ce que se propose ce petit volume qui se penche sur le cas de l'université de Bielefeld, longtemps synonyme de la réforme universitaire outre-Rhin. Réunissant une dizaine de contributions à un colloque tenu à Weimar en 2007, à l'occasion de la parution du premier numéro de la Zeitschrift für Ideengeschichte, il souhaite, selon ses éditeurs, donner une « description dense » d'un milieu universitaire et intellectuel considéré, si ce n'est comme représentatif, du moins comme symptomatique de l'évolution de la société ouest-allemande, lors des trois dernières décennies du XXe siècle. Juxtaposant récits, analyses et témoignages, il propose, à côté d'un simple état des lieux, une approche de la situation intellectuelle d'une société dont l'ambition rappelle le titre d'un célèbre essai de Karl Jaspers, retraçant l'évolution intellectuelle de la société allemande au seuil des années 1930. Loin d'apparaître comme un simple lieu d'échange, l'université de Bielefeld se voit désignée comme la "capitale secrète» du travail de 
réflexion d'une société sur elle-même, offrant par là même, à travers la diversité de ses représentants, une image de l'état d'esprit de la « vieille république rhénane ».

De fait, la création de l'université de Bielefeld s'est inscrite dans une phase d'expansion des institutions universitaires correspondant à l'augmentation de la population estudiantine, accompagnée d'une évolution au moins aussi importante du nombre des enseignants-chercheurs. Bielefeld était en 1969 la troisième institution créée en Rhénanie-Westphalie, après l'université de la Ruhr, à Bochum, et l'Université technique de Dortmund. Hermann Lübbe, qui occupait alors les fonctions de professeur de philosophie à Bochum et de secrétaire d'État à l'Enseignement supérieur à Düsseldorf, retrace la genèse du projet bielefeldois, dans un contexte où les attentes associées à la fondation d'un nouvel établissement se mesuraient déjà à l'aune des expériences récentes. L'université de Bochum avait été conçue comme un établissement réunissant l'ensemble des facultés. Celle de Dortmund marquait un glissement vers un institut universitaire de technologie qui possédait, il est vrai, une longue tradition en Allemagne. Bielefeld devait donc innover par rapport à ces deux modèles, en répondant à la différenciation des fonctions universitaires, tout en évitant ses aspects anomiques. La tâche de définir un tel projet incomba à Helmut Schelsky, alors professeur de sociologie à Münster et directeur d'un institut de recherche à Dortmund, et dont les travaux sur l'évolution des institutions universitaires depuis la réforme humboldtienne avaient connu au début des années 1960 un énorme succès. H. Lübbe retrace les multiples facettes de ce projet, dont un aspect - et pas le moindre - fut que la nomination des membres du comité de fondation fut laissée presque entièrement à l'appréciation de Schelsky, qui souhaitait ainsi pouvoir définir le profil personnel des disciplines de la future université. L'idée dominante fut la création de pôles de recherche, réunissant aussi bien des orientations théoriques spécifiques, telle la théorie du droit pour ne citer qu'un exemple, que des recherches empiriques dans le domaine des sciences sociales en privilégiant les area studies, comme dans le cas des études latino-américaines ou est-européennes. L'élément intégrant devait être la création d'un centre de recherche interdisciplinaire associé à l'université, une création qui répondait à l'échec d'une interdisciplinarité spontanée telle que l'on avait vainement espéré la voir se réaliser dans les grands établissements, débordés par leurs tâches d'enseignement.

La réalisation du projet répondit en grande partie à ces caractéristiques institutionnelles. Un certain nombre des membres du comité de fondation accepta effectivement une charge d'enseignement au fur et à mesure que les bâtiments sortirent de terre. Mais progressivement l'établissement, qui portait désormais le nom de «Reformuniversität ", s'éloignait des prémices idéales de ses débuts, sous la pression des charges d'enseignement et de l'évolution du milieu universitaire au début des années 1970, dont H. Lübbe donne une description mi-amusée, mi-résignée. Pour Helmut Schelsky, rattrapé par son rôle plus qu'ambigu de jeune sociologue sous le IIIe Reich, l'aventure finissait par un retrait de l'université qu'il avait contribué à créer.

L'échec que pouvaient ressentir Schelsky et ceux qui s'étaient engagés à ses côtés s'est en partie matérialisé dans l'évolution qu'a connu le campus. Dès le milieu des années 1970, il occupa un complexe de bâtiments surdimensionné d'un seul tenant, dont le modèle secret est à chercher dans les plans de la Sorbonne du début du XXe siècle. Proposé par un collectif d'architectes, il s'opposait terme à terme à un projet de tours isolées peuplant le terrain à flanc de collines, qui correspondait à la vision initiale de son promoteur. À la place de foyers individualisés, il s'agissait désormais de créer un centre de 
communication, symbolisé dans les premiers plans présentés au ministère par un grand auditorium, ouvert sur un hall central - projet que la réalisation allait transformer en un espace fermé par un grand mur en béton, que des exilés chiliens ont orné quelques années plus tard d'une fresque gigantesque rappelant la répression de la révolution chilienne, objet d'art que seul son classement a sauvé de la destruction. Le bâtiment tel qu'il se présente actuellement dans toute sa fonctionnalité est l'objet d'une analyse de Wolfgang Braungart, qui enseigne la littérature germanique à Bielefeld. Elle se résume en une critique assez sévère de ce qui se présente à l'usager comme une Wissens- und Lernfabrik, bref une usine des savoirs. En comparant l'énorme paquebot, où un dédale de couloirs à n'en pas finir, accessibles seulement par des escaliers froids ou des cages d'ascenseurs, relie les différents départements, bibliothèques et salles de séminaires, avec les projets utopiques des cités éducatives de la première modernité, il conclut sur la vision d'un espace désincarné, où la multiplicité des parcours empêche toute arrivée, comparable à ce " Kein Ort. Nirgends ", à un lieu qui cherche encore son nom.

Lui assigner en revanche une place précise, en rattachant le projet à un moment plus que spécifique de l'histoire allemande, tel est l'objectif de la contribution de Clemens Albrecht qui se tourne vers le "cas Schelsky", en l'interprétant comme l'expression d'une " conversion par adaptation ", excluant toute prise de distance par rapport aux réalités sociales. S'appuyant sur un dialogue entre Helmut Schelsky et Friedrich H. Tenbruck sur la finalité de toute réforme universitaire, il esquisse la critique d'une perception du social qui s'accommode de l'idée que l'on se fait de la réalité en s'y conformant. Schelsky et son projet d'une université de réforme $\mathrm{y}$ fait figure d'une «hypostasierte Realitätsanpassung ", préparant le terrain d'un enseignement réduit à sa technicité rationnelle, en admettant que la crise du moment sera la normalité de demain.

Fruit d'une "conversion par passage d'un système à l'autre", caractéristique de la génération de l'immédiat après-guerre, la Reformuniversität marquera ainsi la naissance d'un modèle de "modernisation adaptive" que Cl. Albrecht qualifie de "BielefeldSyndrom» et, pour parler plus radicalement, d'«une pathologie spécifique à la République fédérale ». Enfin, pour compléter le tableau, Niklas Luhmann, que Schelsky avait proposé pour la chaire de sociologie théorique à Bielefeld, apparaît ici en tant que témoin à charge, décrivant et analysant l'institution universitaire en termes d'organisation, accréditant l'idée de la Betriebsmässigkeit (opérationnalité) du fonctionnement des établissements d'enseignement et de recherche.

Faut-il parler de généralisation abusive ou de règlement de compte entre sociologues? Méthodologiquement, la démarche que propose $\mathrm{Cl}$. Albrecht correspond certes bien à ce que le sous-titre de la collection d'essais nomme une «ideengeschichtliche Nachfrage ", c'est-à-dire une enquête idéologique plus ou moins compréhensive.

Plus modéré est le témoignage de Hartmut von Hentig, en tant qu'inspirateur et acteur de la réforme pédagogique, associée à la création d'une Laborschule qui devait en même temps faire fonction d'école préparatoire, intégrée au campus universitaire. Sur le ton du regret de ne pas avoir atteint tout ce qui aurait été possible, H. v. Hentig décline le Bielefeld-Syndrom en termes de compromis douloureux, rendant la réforme «administrable» au point de la compromettre. Décrire ce que pouvait être l'inspiration et le but pragmatique de la réforme pédagogique de $\mathrm{H}$. v. Hentig est l'objet de la contribution suivante, très documentée, de Jürgen Oelkers. Comparant les projets défendus aux États-Unis au début du XXe siècle par John Dewey et Robert Maynard 
Hutchins, il décrit, en prenant appui sur l'exemple du Chicago University College, l'horizon pragmatique d'une réforme qui, à Bielefeld, n'a que très partiellement réussi.

La réussite fut en revanche un sort largement partagé par le département de sociologie et la faculté des sciences historiques, à laquelle on allait accoler, vers la fin des années 1970, le nom d' "école de Bielefeld ». C'est à cette dernière que Frank Becker consacre une brève étude, sous le titre provocateur "Mit dem Fahrstuhl in die Sattelzeit?», qui parodie celui d'un essai populaire sur la visite archéologique des sous-sols romains de la vallée rhénane. Comme l'indique le sous-titre, il se tourne pour l'essentiel vers le rôle qu'ont joué Reinhart Koselleck et Hans-Ulrich Wehler dans l'émergence du profil intellectuel de la faculté . Mettant en avant les différences d'origine, de formation et de style des deux hommes, F. Becker souligne leur convergence sur quelques points essentiels, telle l'idée d'une rupture constitutive de la modernité et la préférence d'une approche systématique, s'opposant à toute narration incontrôlée. L'analyse, qui décrit un Koselleck individualiste et un Wehler particulièrement combatif, laisse à peine entrevoir une situation de dialogue qui impliquait de nombreux autres acteurs et qui était aux yeux de beaucoup, surtout ceux ou celles venant de l'extérieur, la véritable marque de la faculté. C'est cette ouverture qui favorisa l'intégration de savants venant d'ailleurs, comme Sidney Pollard, et le renouvellement des thèmes de recherche, dont le caractère collectif justifiait en grande partie l'hétéro-désignation d'« école de Bielefeld ». Beaucoup plus distancés restaient les rapports entre historiens et sociologues, malgré la forte préférence de l'école dite de Bielefeld pour l'histoire sociale. Les deux analyses de Markus Krajewski et Jürgen Kaube, consacrées à l'œuvre de Niklas Luhmann, se concentrent de façon exemplaire sur ses méthodes de travail et les caractéristiques épistémologiques de la théorie des systèmes. Il aurait été instructif de confronter ici plus explicitement les différentes conceptions du recours à la théorie, que Reinhart Koselleck avait revendiqué dès 1972 pour l'historiographie.

Cela paraît d'autant plus important que le jugement que portent les différentes contributions sur ce qu'a été et aurait pu être Bielefeld est le plus souvent l'expression d'orientations théoriques plus ou moins explicites. C'est surtout le cas des deux rapports s'attachant au vécu d'une immersion dans l'univers bielefeldois, et que donnent Gustav Seibt et Valentin Groebner. G. Seibt décrit pour l'essentiel son expérience des séminaires de Koselleck et de Luhmann au cours du semestre d'hiver 1983-1984, marqué par le débat autour du déploiement des fusées Pershing sur la frontière à l'Est. Ce qui caractérisait les deux expériences fut apparemment la pesanteur ressentie de la théorie de la continuité chère à Löwith, revue par Koselleck et Blumenberg d'un côté, et le jeu lumineux avec la leggerezza et pesantezza d'Italo Calvino entrant dans le rapport à un monde lourd de catastrophes, présenté par Niklas Luhmann de l'autre, le tout étant attisé par l'interprétation que donnait au même moment Karl Heinz Bohrer (qui représentait depuis 1982 l'histoire littéraire à Bielefeld) de Salammbô de Flaubert, lu comme l'expression d'une autonomie esthétique au-delà de toute considération morale et philosophique... V. Groebner, quant à lui, nous fait part non sans arrogance de son expérience de séances d'orientation théorique, dans lesquelles il croit reconnaître un des instruments d'une autoreprésentation qui, par un jeu de citations plus ou moins codé, permettait aux acteurs de la scène bielefeldoise de prendre conscience d'eux-mêmes. L'expérience bielefeldoise se solde pour cet observateur par un constat qui coïncide avec l'énoncé du titre du volume, en attribuant à Bielefeld le passé simple qui convient à un moment de l'histoire. 
À regarder l'ensemble des contributions, on est tenté de se référer à des ouvrages comme celui de Karl Jaspers, à la limite au Georg Lukacs de La Destruction de la raison, ou à Karl Kraus, références qui toutes s'avèrent finalement inappropriées. Afin de restituer les courants qui ont porté le Bielefeld-Syndrom, pour reprendre l'expression de $\mathrm{Cl}$. Albrecht, l'enquête que propose le titre collectif aurait dû sans doute se pencher davantage sur le réseau complexe créé sous l'impulsion de Helmut Schelsky, autour du comité de fondation. Des lieux universitaires comme Münster, avec le philosophe Joachim Ritter et le sociologue Hans Freyer, ou Heidelberg où enseignaient des historiens comme Werner Conze et Erich Maschke et où le Alfred-Weber-Kreis et l'Arbeitskreis für Sozialgeschichte, avec des chercheurs comme Reinhart Koselleck et Jürgen Kocka, ont joué un rôle déterminant dans le développement d'une histoire sociale et culturelle moderne, constituaient des foyers intellectuels importants. Non moins importante a été l'influence de places comme Cologne ou Bochum. À Cologne, une des universités recréées sous la République de Weimar en 1919, Theodor Schieder exerçait une grande influence, tandis que Richard Konetzke et son élève Günther Kahle y représentaient l'histoire latinoaméricaine, appelée à jouer un rôle primordial dans les area studies de Bielefeld. Parmi les jeunes chercheurs, on y comptait les historiens Christian Meier, Hans-Ulrich Wehler et le linguiste Harald Weinrich qui allaient, tous les trois, jouer un rôle important dans le développement du projet bielefeldois. De Bochum sont venus Rudolf Vierhaus et Max Imdahl, respectivement historiens du monde moderne et de l'histoire de l'art contemporain. De Constance enfin, l'influence de Hans-Robert Jauß et Wolfgang Iser, aux noms desquels il faut associer le cercle «Poetik und Hermeneutik », s'est exercée sur la conception de la faculté de lettres et de linguistique («Lili») de Bielefeld, sans oublier dans cet ensemble de lieux, d'hommes et d'institutions couvrant plusieurs générations, les nombreux chercheurs qui, comme Jürgen Kocka, ont abordé l'histoire du prolétariat, comme Hans-Ulrich Wehler, celle de l'impérialisme, ou comme le groupe qui s'était constitué à Heidelberg autour de Reinhart Koselleck, celle des camps et de la société postconcentrationnaire. C'est ce vaste ensemble plus qu'hétérogène qui permettrait en définitive de prendre la mesure de la place de cette tentative de réforme si singulière dans le paysage intellectuel de la République fédérale, au cours des trois dernières décennies du XXe siècle. Ce ne sera pas le moindre mérite de cette collection d'essais plutôt provocants que de susciter une telle entreprise ! 